\title{
Correlación entre velocidad y comprensión lectora en alumnos de educación primaria, Kusu Chapi, región Amazonas
}

\section{Correlation between speed and reading comprehension in primary school students, Kusu Chapi, Amazonas region}

\author{
Abiezer Chuintam Sanchez" ${ }^{1}$ José Darwin Farje Escobedo²
}

\begin{abstract}
RESUMEN
El presente trabajo tuvo como objetivo general determinar la correlación entre velocidad lectora y comprensión lectora en alumnos de quinto grado de educación primaria de la Institución Educativa 17347 de la comunidad de Kusu Chapi, distrito de Imaza, provincia de Bagua, región Amazonas, Perú. La investigación fue descriptiva, con diseño descriptivo correlacional. La muestra estuvo conformada por 20 alumnos. Los instrumentos utilizados para la medición de la velocidad lectura fueron una ficha de registro que permitió medir la cantidad de palabras leídas por minuto, a partir de una lectura; y para el nivel de comprensión lectora, una prueba de opción múltiple, conformada por diez preguntas, cinco de tipo literal y cinco de tipo inferencial. Según los resultados, el 70\% de estudiantes en promedio, se ubican en el nivel bajo de comprensión lectora en los niveles literal e inferencial; además, el 85\% tiene un nivel deficiente en la velocidad lectora; y finalmente, al comparar los hallazgos obtenidos entre el nivel de comprensión lectora y su relación con la velocidad lectora, se concluye que la comprensión lectora no es independiente del nivel de velocidad lectora de los alumnos del quinto grado, con el 95\% de confianza; lo que significa que los estudiantes tienen una deficiente velocidad para leer y en consecuencia, no comprenden los textos que leen.
\end{abstract}

Palabras clave: Comprensión lectora, velocidad lectora

\begin{abstract}
The present work had as general objective to determine the correlation between reading speed and reading comprehension in fifth grade students of primary education of the Educational Institution 17347 of the Kusu Chapi community, Imaza district, Bagua province, Amazonas región, Peru . The research was descriptive, with a correlational descriptive design. The sample consisted of 20 students. The instruments used to measure the reading speed were a record card that made it possible to measure the number of words read per minute, based on one reading; and for the level of reading comprehension, a multiple-choice test, consisting of ten questions, five of a literal type and five of an inferential type. According to the results, $70 \%$ of students on average are located at the low level of reading comprehension in the literal and inferential levels; Furthermore, 85\% have a poor level of reading speed; and finally, when comparing the findings between the level of reading comprehension and its relationship with reading speed, it is concluded that reading comprehension is not independent of the level of reading speed of fifth grade students, with $95 \%$ of confidence; which means that students have a poor speed to read and consequently do not understand the texts they read.
\end{abstract}

Keywords: Reading comprehension, reading speed

\footnotetext{
Bachiller en Educación egresado de la Facultad de Educación y Ciencias de la Comunicación de la Universidad Nacional Toribio Rodríguez de Mendoza de Amazonas.Correo electrónico: abiezer.cs@gmail.com

${ }^{2}$ Licenciado en Educación Primaria, Docente Asociado, Universidad Nacional Toribio Rodríguez de Mendoza de Amazonas. Correo electrónico:jose.farje@untrm.edu.pe
} 


\section{INTRODUCCIÓN}

Con mayor frecuencia, la sociedad casi en su conjunto, tiene al frente, sucesos, eventos o acontecimientos que a lo mejor, no es capaz de dar respuestas atinadas o acertadas, que algunos los llaman incertidumbres, que suelen dejar perplejas a las personas, en los países desarrollados, pero también en los subdesarrollados; esa imposibilidad para enfrentar retos que aparece en su camino; y para la generalidad de individuos, la educación debe preparar a la humanidad para enfrentar desafíos; Campos (2005), dice que "la mejora de la educación exige, de modo indiscutible, repensar modelos que tienen como responsabilidad, la formación profesional del profesorado; para el efecto, debe demandar la participación y colaboración de la sociedad dentro y fuera de la escuela".

También se percibe una intranquilidad, preocupación y en muchos casos indiferencia por la enseñanza de la lectura, que desde cualquier punto de vista, constituye una complejidad; realidad que no debe causar extrañeza, sobre todo en las zonas donde hay una serie de necesidades insatisfechas; al respecto, Mora (2020), refiere que "leer significa activar un amplio arco cognitivo que involucra la curiosidad, la atenci $\mathrm{n}=1$ aprendizaje y la memoria, la emoción, la consciencia y el conocimiento. Es quizás el mejor medio para construir un puente definido entre humanidades y ciencia".

Si el aprendizaje de la lectura, implica toda una complejidad en términos de procesos cognitivos y didácticos, que activa dimensiones del ser humano, y que existe una gran diferencia con el lenguaje oral, la lectura, por el contrario, carece de una base genética; entonces, siendo un tanto enérgicos y realistas, se puede afirmar que, probablemente los docentes no estamos preparados para enseñar tamaña competencia a los escolares. "La escuela, al parecer, no entiende que sus exiguos e insuficientes resultados, en cuanto a la formación de lectores y escritores, sin lugar a dudas, ocasionan o generan, precisamente exclusiones de gran envergadura, que, en definitiva, influyen plenamente en la ciudadanía" (Cassany, 1994 y Esté 1999).

En el caso particular peruano, la cultura letrada está cada vez más lejos de los escolares y en general de toda la familia, el acto de leer, que hace algunas décadas era actividad familiar y escolar de práctica constante. En el escenario escolar, se aprecia que los escolares leen mal, porque los profesores también leen mal y enseñan mal, la lectura inicial; Cueto (2001), nos dice que, "el maestro de primaria pierde la valiosa oportunidad de direccionar al niño en la lectura como proceso lógico, placentero y significativo; al no reforzar su comprensión personal cuando está leyendo un texto contribuyendo a la incomprensión del valor práctico de la lectura".
Por su parte, Díaz (2014), dice que, "la adquisición de la habilidad lectora en el Perú, de todas maneras, deja mucha que desear; y el esfuerzo del Estado para revertir eso, a veces no permite apreciar resultados optimistas; y toda esa realidad, condiciona el rendimiento académico en todas las materias".

Por otro lado, la velocidad lectora, está relacionada directamente a la comprensión, si un escolar lee un texto con fluidez, claridad y rapidez, entonces hay mayores posibilidades que comprenda lo que está leyendo; al respecto, Nuttall (2006), afirma que, "la frustración que produce el leer lentamente, los lectores que no entienden un material a menudo disminuyen la tasa de lectura y en consecuencia, no les gusta leer porque les toma mucho tiempo; y a la larga, van perdiendo el sentido por leer".

La situación problemática descrita, de todas maneras, es aún más complicada en la zona selvática de la región Amazonas, donde están presentes etnias Awajún y Wampis y el reto del docente es enseñar en lengua materna y segunda lengua. Al respecto, el Ministerio de Educación (2014) del Perú, publicó los resultados de los escolares Awajún en comprensión lectora en educación primaria; y "el 56\%, se encuentra en inicio, y solamente el $18 \%$ está en el nivel satisfactorio; y además, no lograban alcanzar el nivel de comprensión crítica; esto nos invita a la reflexión, dado que los alumnos presentan dificultades significativas para leer textos y el problema persiste durante toda su escolaridad".

Es muy conocido que últimamente los procesos orientados a la adquisición de la lectura, de todas maneras, están enfrentando barreras y obstáculos que no permiten su aprendizaje de manera significativa, a pesar que constituye una actividad humana posibilita adquirir conocimientos y desarrollar capacidades y competencias en diferentes contextos y realidades; constituyendo, lo descrito, la justificación principal del estudio.

Murcia, Torres y Velasco (2018), realizaron una investigación sobre comprensión lectora, en estudiantes de la Institución Educativa departamental integrada de Sutatausa sede Novoa. El propósito fue determinar el nivel de comprensión lectora en su nivel literal y aplicar una secuencia de actividades didácticas; concluyendo que para mejorar los resultados obtenidos, que muestran deficiencias en el nivel de comprensión literal, el planteo una secuencia de actividades didácticas que respondieron a los intereses de los estudiantes, aprovechando sus características y particularidades, en un contexto de relevancia para los alumnos; mejoraron de manera significativa la comprensión.

Bonilla, Durón y Fúnez (2015), estudiaron la velocidad y la comprensión lectora; su objetivo fue conocer la velocidad y comprensión lectora de estudiantes y concluyeron que la comprensión lectora 
obtenida para la muestra de estudio fue de $67 \%$, segmento que se encontraba en el nivel medio; los estudiantes desarrollaron mayor velocidad lectora en lecturas de libros que los obtenian con facilidad y que era de su interés; resultados que tiene relevancia, dado que la comprensión es fundamental para optimizar el aprendizaje y el rendimiento académico; si aumenta la variable velocidad, aumenta la comprensión, por lo que la lectura eficaz o veloz, se corresponde con la comprensión del texto leído, se complementan de manera activa, a mayor velocidad lectora, mayor comprensión lectora.

Huamán (2018), en su estudio sobre la atención y la velocidad lectora en escolares de la Institución Educativa Bellavista, concluyó que existe una gran similitud relevante entre la atención selectiva, atención sostenida y atención dividida y la velocidad lectora y agilidad lectora.

El estudio de Villasante (2018) sobre la fluidez lectora en estudiantes de segundo grado de las instituciones educativas del nivel primario 70581, Acomocco y 80621 de Juliaca, concluye que a un nivel de significancia del 5\% los estudiantes, presentan diferencia significativa en cuanto a la fluidez lectora con un valor de significancia de 0,010 $($ sig< $<.05)$; los problemas de fluidez lectora de los niños se presenta por repetición de palabras, mala pronunciación de letras, omisiones y sustituciones, lo que ocasiona que la lectura sea deficiente, por tal motivo la comprensión lectora presenta niveles bajos; las deficiencias en fluidez y comprensión no favorecen los procesos de evolución del lenguaje y comunicación de los niños, quienes presentan un bajo dominio de comprensión, eso dificulta los procesos de aprendizaje en el periodo inicial de educación general básica.

También se hace mención a la investigación local, de Gómez (2019), sobre el nivel de comprensión lectora en los relatos populares, en quinto grado de la Institución Educativa n. ${ }^{\circ} 18107$ de Yambrasbamba, las conclusiones fueron que el nivel de comprensión lectora en la dimensión literal antes de la selección, aplicación y evaluación de los relatos populares está en un nivel malo; luego de la selección, aplicación y evaluación de relatos se encuentra en un nivel de regular y bueno. El nivel de comprensión en la dimensión inferencial, se encuentra en un nivel regular; luego de la selección, aplicación y evaluación de estos relatos se encuentra en un nivel excelente.

A continuación, es pertinente ocuparnos de los fundamentos teóricos de la velocidad lectora; para López (2013), es "la capacidad propia del ser humano, para reconocer las palabras de forma automática y rápida, la velocidad lectora es un indicador, en concreto, de la fluidez y de todas maneras nos conlleva hacia la comprensión lectora". En esa perspectiva, se puede agregar que: "Las personas que están leyendo bien y que tienen un buen vocabulario leerán más, y aprenderán más significados; mientras que, aquellos lectores que demuestran tener vocabularios inadecuados, que además demuestran leer despacio, tienden a leer menos; lo que tributa a que ellos tengan un desarrollo más lento y un pobre vocabulario; dicho de otro modo, inhibe el desarrollo de su habilidad lectora" (Puente, 1991).

Siguiendo el pensamiento de Fry (1995), distingue tres niveles de velocidad lectora, según el tipo que realizan; "las lectura corriente y común", es aquella lectura que suelen utilizar para leer revistas, libros y textos que tienen una categoría de fáciles o sencillos. Normalmente se desarrolla a una velocidad de 250 a 300 palabras por minuto (ppm) y con un nivel comprensivo que alcanza al $70 \%$ aproximadamente. También considera las "lectura de estudio", que se caracteriza por ser lenta, este caso particular de lectura es empleada normalmente con materiales más fáciles; además, exige un alto nivel de concentración y atención; donde resulta ser muy importante que se garantice un nivel alto de comprensión; por consiguiente, se desarrolla a una velocidad de entre 180 a 200 palabras por minuto (ppm); su propósito es alcanzar un nivel comprensivo de aproximadamente $90 \%$; y, finalmente, "la lectura superficial", en realidad, este tipo de lectura es utilizada para explorar textos, el promedio comprensivo es de $50 \%$ y su velocidad; se destaca además que, alcanzan a leer hasta 800 palabras por minuto".

En el caso particular de los estudiantes de educación básica, y en específico los de educación primaria, en el quinto y sexto grado, el promedio de velocidad de lectura adecuada es de 110 palabras por minuto, para garantizar comprensión literal, (García, s/f).

La actividad lectora exige pasar por momentos o etapas específicas que, en la idea de Solé (2004), refiere que son tres: un primer momento que lo denomina antes de la lectura, "donde el lector debe cuestionarse para qué va a leer, se puede decir que en este subproceso o su etapa, el lector tendrá muy en cuenta objetivos asociados de la lectura, como base consideraciones tales como: para aprender, para mejorar la práctica de la lectura en voz alta, para desarrollar hábitos de un buen lector, para indagar lo que ha considerado el autor dentro del escrito, para hacer predicciones dentro de un texto". Asimismo, la autora propone un segundo momento; "donde la persona hace gala de su experticia o pericia en la lectura; paralelamente al acto de leer, el lector puede resumir el texto, también puede enunciar o formular preguntas que se van gestando a lo largo del texto, además puede aclarar dudas sobre lo que lee, practicando la relectura; es importante, en esta etapa hacer uso de diccionario, y de igual manera recrear imágenes sobre lo que va leyendo".

Finalmente, Solé (2004), refiere un tercer momento, 
donde el "lector está en condiciones de construir resúmenes más elaborados y sistematizados, comentar lo leído a sus pares u otras personas, diseñar bosquejos gráficos, recontar lo que ha leído; pero también hay que mencionar que es capaz de responder y formular preguntas" (pág. 128).

En lo que respecta a la definición de comprensión lectora, Antezana (2009), menciona que "la comprensión lectora corresponde a un proceso donde se va a construir significados, relacionados de todas maneras a los aportes del texto y que depende en gran medida, del conocimiento que tiene el lector; vale decir, el lector es protagonista en la reconstrucción de significados".

En cuanto a los niveles de comprensión lectora, Sánchez (2001), refiere que son tres los niveles, "el literal (se relaciona a identificar las ideas principales, explícitas de un texto); inferencial (permite que el lector establezca relaciones en lo que lee, identificar elementos implícitos del texto) y criterial (cuando el lector argumenta expresando acuerdo o desacuerdo con el texto)"; otros como Pinzas (1997), fundamenta los niveles clásicos en los que sobresale la comprensión metacognitiva que se relaciona con crear espacios asociados a la reflexión sobre lo que va comprendiendo en lector, en relación al texto que lee (Solé, 1994); además, Smith (1998), asiente que la comprensión literal implica el reconocimiento de las ideas principales y las secundarias, considerando el orden de las acciones o los hechos que ocurren, el tempo y también los lugares, con el dominio de un lenguaje básico.

Ahora bien, al hacer referencia al nivel de comprensión inferencial, Condemarín (1999) sostiene que el lector es capaz de asociar lo leído a su experiencia personal y a partir de ella, realizar conjeturas e hipótesis relacionados al texto. Además, el lector puede realizar inferencias sobre detalles adicionales; dicho de otro modo, el individuo que lee también es capaz de realizar inducciones de significado o enseñanza moral a partir de la idea principal. Para Smith (1998), el lector, es capaz de extraer las ideas y mensajes que no están explícitas en el texto; además de construir, cimentar o deducir significados o interpretaciones propias.

Sánchez (2001), hace referencia al nivel crítico, denominado también criterial, que se asocia directamente a que el lector toma posición o postura frente a lo que dice el autor y lo integra con lo que él sabe.

\section{MATERIAL Y MÉTODO}

La investigación fue descriptiva correlacional. La muestra lo conformaron 20 alumnos de la institución educativa 17347 de Kusu Chapi, distrito Imaza, provincia Bagua, región Amzonas, Perú. El muestreo fue intencional.

Los instrumentos utilizados para la medición de la velocidad lectura fue una ficha de registro que permitió medir la cantidad de palabras leídas por minuto, a partir de una lectura; y para el nivel de comprensión lectora, una prueba de opción múltiple, conformada por diez preguntas, cinco de tipo literal y cinco de tipo inferencial.

\section{RESULTADOS}

\section{Figura 1}

Nivel de comprensión lectora en la dimensión literal.

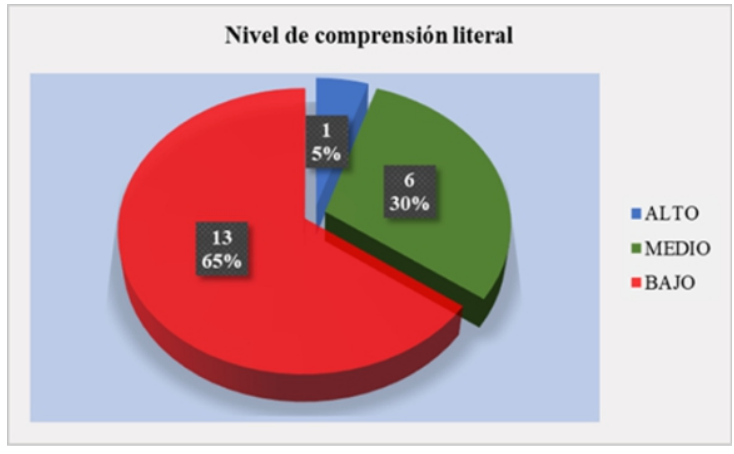

La figura 1, muestra que el $65 \%$ tiene nivel bajo de comprensión lectora en la dimensión literal, el 30\% tiene nivel medio y solamente el $5 \%$ se ubica en el nivel alto.

\section{Figura 2}

Nivel de comprensión lectora en la dimensión inferencial.

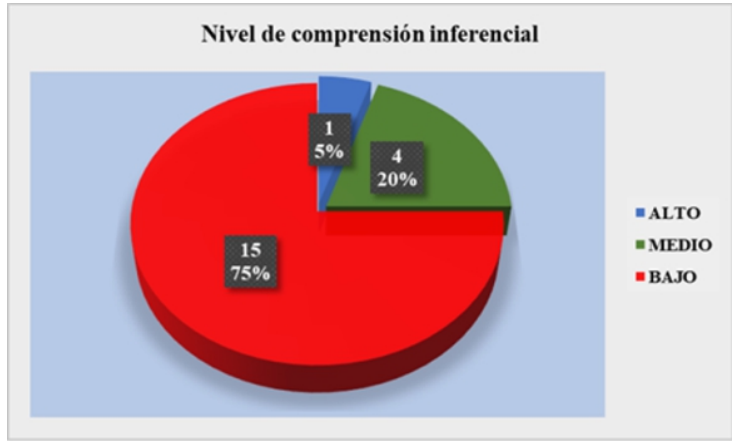

La figura 2, presenta los resultados obtenidos en el nivel de comprensión inferencial, el $75 \%$ está en el nivel bajo, el $20 \%$ está en el nivel medio y el $5 \%$ en un nivel alto. 


\section{Figura 3}

Nivel de comprensión lectora de los estudiantes.

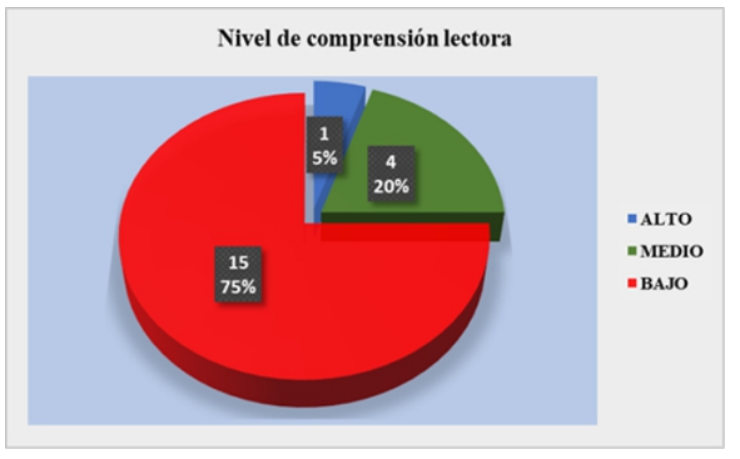

La figura 3, respecto al nivel de comprensión lectora de los estudiantes, permiten percibir las grandes debilidades para comprender textos, dado que el 5\% tiene un nivel alto, frente a un nivel bajo del $75 \%$, que no comprende los textos que leen.

\section{Figura 4}

Porcentaje del nivel de velocidad lectora

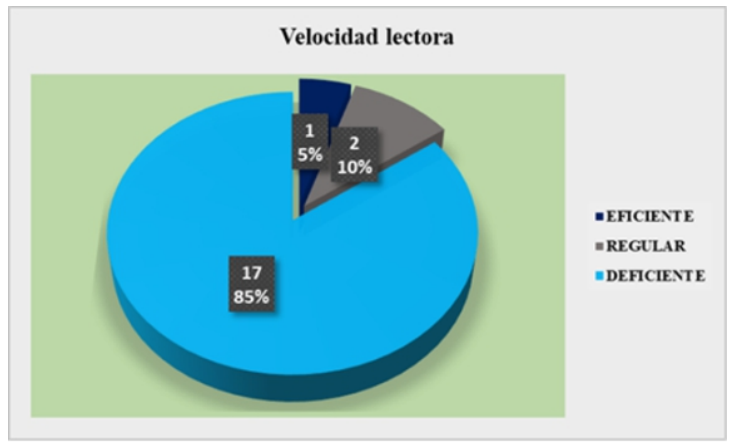

La figura 4, muestra que el $85 \%$ de los estudiantes tienen un nivel de velocidad lectora es deficiente, el $10 \%$ alcanzan el niveles de regular y un 5\% tiene un nivel eficiente.

\section{Tabla 1}

Análisis estadístico de la comprensión y velocidad lectora de los alumnos

\begin{tabular}{lcccccccc}
\hline COMPRENSIÓN & \multicolumn{2}{c}{ EFICIENTE } & \multicolumn{2}{l}{ REGULAR } & \multicolumn{2}{l}{ DEFICIENTE } & \multicolumn{2}{c}{ TOTAL } \\
\cline { 2 - 9 } \multicolumn{1}{c}{ LECTORA } & $\mathbf{N}^{\circ}$ & $\%$ & $\mathbf{N}^{\circ}$ & $\%$ & $\mathbf{N}^{\circ}$ & $\%$ & $\mathbf{N}^{\circ}$ & $\%$ \\
\hline ALTO & 1 & 5.0 & 0 & 0.0 & 0 & 0.0 & 1 & 5.0 \\
MEDIO & 0 & 0.0 & 2 & 10.0 & 2 & 10.0 & 4 & 20.0 \\
BAJO & 0 & 0.0 & 0 & 0.0 & 15 & 75.0 & 15 & 75.0 \\
\hline TOTAL & $\mathbf{1}$ & $\mathbf{5 . 0}$ & $\mathbf{2}$ & $\mathbf{1 0 . 0}$ & $\mathbf{1 7}$ & $\mathbf{8 5 . 0}$ & $\mathbf{2 0}$ & $\mathbf{1 0 0 . 0}$ \\
\hline
\end{tabular}

$$
X_{C}^{2}=28,824 \quad \mathrm{p}=0,000
$$

\section{Pruebas de chi-cuadrado}

\begin{tabular}{crcc}
\hline \hline & Valor & g1 & $\begin{array}{c}\text { Sig. asintótica } \\
\text { (bilateral) }\end{array}$ \\
\hline Chi-cuadrado de Pearson & $28,824^{a}$ & 4 &, 000 \\
Razón de verosimilitudes & 15,182 & 4 &, 004 \\
N de casos válidos & 20 & & \\
\hline \hline
\end{tabular}

a. 8 casillas $(88,9 \%)$ tienen una frecuencia esperada inferior a 5 .

La frecuencia mínima esperada es, 05 .

La tabla 5; se observa que, $\mathrm{p}=0,000<0.05$, se rechaza la Ho (hipótesis nula), concluyendo que la comprensión lectora no es independiente del nivel de velocidad lectora de los alumnos, con el $95 \%$ de confianza.

\section{DISCUSIÓN}

A partir de los hallazgos, sobre la comprensión lectora y su relación con la velocidad lectora, de los estudiantes que cursan el quinto grado de educación primaria en la I.E. 17347 de Kusu Chapi; se puede señalar que existen dificultades para comprender textos que leen; en esa dirección, Serna y Díaz (2015), sostienen que, el ejercicio de la comprensión lectora, de todas maneras, es una práctica cotidiana e imperativa en la configuración del mundo contemporáneo, y que, indiscutiblemente, debe ser desarrollada desde los primeros años de la escolaridad.

Analizando los resultados asociados al nivel de comprensión lectora literal e inferencial, se percibe que los estudiantes presentan dificultades significativas para comprender textos que leen, el $70 \%$ en promedio, obtienen un nivel bajo en la comprensión lectora; encontrando similitudes con la investigación de Murcia, Torres y Velasco (2018), en su estudio de la comprensión lectora en el nivel literal, concluyen que los escolares muestran deficiencias en el nivel de comprensión literal, por lo que, plantearon un conjunto de actividades en base a los intereses y preferencias de los escolares y finalmente, mejoraron de manera significativa la comprensión de textos.

De igual manera, también se coincide con los resultados de Villasante (2018), en su estudio sobre fluidez lectora en estudiantes de segundo grado, concluye que, la falta de fluidez ocasiona que la lectura sea deficiente, por lo que la comprensión lectora presenta niveles bajos. También existe una relativa similitud con el estudio de Gómez (2019), cuando estudia el nivel de comprensión en estudiantes de educación primaria, concluye que los niveles de comprensión literal e inferencial de los escolares, se encuentra entre malo y regular.

Haciendo el análisis de los resultados obtenidos sobre la velocidad lectora de los escolares, se percibe que 
presentan dificultades o conflictos para leer textos con una velocidad necesaria que garantice la comprensión; el 85\% está en el nivel deficiente, la velocidad de su lectura es muy lenta, en función a su edad y grado de escolaridad (quinto grado); encontrando cierta similitud con el estudio de Bonilla, Durón y Fúnez (2015), en su estudio sobre la velocidad y comprensión, concluye que, en general, la comprensión lectora es deficiente; sin embargo, cuando los escolares practican la lectura de libros que los obtienen con cierta facilidad y es de su interés, tienden a incrementar su velocidad lectora.

Relacionando las variables de comprensión lectora y velocidad lectora, según los resultados, teniendo en cuenta que, $\mathrm{p}=0,000<0.05$ se rechaza Ho, concluyéndose que la comprensión lectora no es independiente del nivel de velocidad lectora de los alumnos, con el 95\% de confianza; lo que significa que si los estudiantes tienen una deficiente velocidad para leer, entonces, no van a comprender lo que leen.

Se ha encontrando coincidencias con el estudio de Huamán (2018); sobre la atención y la velocidad lectora en escolares; donde concluye que existe similitud relevante entre lectura selectiva, velocidad lectora y agilidad lectora; Asimismo, se encuentra mayor similitud con el estudio de Bonilla, Durón y Fúnez (2016), en su estudio sobre velocidad y comprensión lectora, quienes concluyeron que, si aumenta la variable velocidad también aumenta la comprensión de textos, por lo que la lectura eficaz o veloz, se corresponde con la comprensión del texto leído, se complementan de manera activa, a mayor velocidad lectora, mayor comprensión lectora.

En la presente investigación se ha logrado percibir que los estudiantes awajún, practican la lectura de manera muy lenta, descifran con lentitud los fonemas, las sílabas, las palabras y las frases.

\section{CONCLUSIONES}

El nivel de desarrollo de la comprensión lectora en estudiantes de quinto grado de educación primaria de la Institución Educativa 17347 de la comunidad awajún de Kusu Chapi, distrito Imaza, es deficiente, el $65 \%$ alcanza el nivel bajo en la comprensión literal; y el $75 \%$, alcanza ese mismo nivel en la comprensión inferencial.

El $85 \%$ de los estudiantes tienen un nivel de velocidad lectora deficiente, el $10 \%$, regular y solamente del $5 \%$ es eficiente; es decir, la velocidad lectora de los estudiantes es tan lenta que no asegura la comprensión de los textos que leen.

Al comparar los resultados obtenidos entre el nivel de comprensión lectora y su relación con la velocidad lectora, se concluye que la primera no es independiente de la segunda.

\section{REFERENCIAS BIBLIOGRÁFICAS}

Antezana, L. (2009). Teorías de la lectura. Altiplano.

Bonilla, J., Durón, W. y Fúnez, M. (2015). La velocidad y la comprensión lectora (tesis de pregrado). Universidad Pedagógica Nacional Francisco Morazán de Tegucigalpa, Honduras. https://metodologiadeinvestigacioncuantitativ a.files.wordpress.com/2015/12/velocidad-ycomprensic $3 b 3 n$-lectora.pdf

Camps, A. (7 de enero de 2005). La comprensión lectora, problema de todos. Diario El País, p. 2. https:/elpais.com/diario/2005/01/07/socieda d/1105052411_850215.html

Cassany, D. (1994). Enseñar lengua. Graó.

Condemarín, M. (1999). Lectura temprana. Andrés Bello.

Cueto (2001). Oportunidades de aprendizaje y rendimiento en comunicación. Grade.

Díaz, J. (2014). Educación inicial y rendimiento en la escuela. Grade.

Escurra, L. M. (1988). Cuantificación de la validez de contenido por el criterio de jueces. Revista de Psicología de la Pontificia Universidad Católica del Perú 6(1 y 2), 103-111.

Esté, A. (1999). El aula punitiva. Publicaciones UCAB.

Fry, E. (1995). Ejercicios de lectura para velocidady comprensión. Jamestown Editores.

García, P. (s/f). Prueba de caracterización del nivel de fluidez y comprensión lectora de los estudiantes de quinto grado. Instituto Colombiano para la Evaluación de la Educación. https://iedjesusdenazaret.files .wordpress.com/2017/06/anexo-2-pruebalectura-quinto-grado-1.pdf

Gómez, L. (2019). Nivel de comprensión lectora en los relatos populares, en quinto grado de primaria de la Institución Educativa n. ${ }^{\circ} 18107$ 
de Yambrasbamba. http://repositorio. untrm.edu.pe/bitstream /handle/ UN TRM /2044/G\%c3\%b3mez\%20 Vargas \% 20Luis.pdf? sequence $=1 \&$ isAllowed $=y$

Huamán, V. (2018). La atención y la velocidad lectora en niños de segundo grado de la Institución Educativa Bellavista. [Tesis de posgrado, Universidad César Vallejo. https://repositorio.ucv.edu.pe/bitstream/handl e/20.500.12692/25601/Huaman_MV.pdf?seq uence $=1$ \&isAllowed $=\mathrm{y}$.

López, C. (2013). Propuesta de estrategias y metodologías para mejorar la fluidez lectora en los estudiantes de tercer año básico de la ciudad de Villa del Mar. El Agóra.

Mora, F. (2020). Neuroeducación y lectura. De la emoción a la composición de las palabras. Alianza.

Nuttall, C. (1996). Enseñar habilidades de lectura. Teaching.

Ministerio de Educación (2014). Cómo mejor la comprensión lectora de nuestros estudiantes de primaria EIB. http://umc.minedu.gob.pe /wp-content/uploads/2015/02/4to-EIB2013_Awajun-BAJA.pdf

Ministerio de Educación (2020). Resolución Viceministerial $\mathrm{n} .{ }^{\circ}$ 133-2020-Minedu. https:// cdn.www.gob.pe/uploads/document/file/101 6161/RVM_N_133-2020-MINEDU.pdf

Murcia, S., Torres, A. y Velasco, M. (2018). La comprensión lectora en el nivel literal, en estudiantes del grado tercero de la Institución Educativa Departamental Integrada de Sutatausa sede Novoa. https://repository.ucc .edu.co/bitstream/20.500.12494/6553/1/2018 _comprension_lectora_nivel.pdf

Pinzas, J. (1997). Metacognición y lectura. Pontificia Universidad Católica del Perú.

Puente, A. (1991). Comprensión de la lectura y acción docente. Pirámide

Sánchez, C., Reyes, C. y Mejía, K. (2018). Manual de términos en investigación cientifica, tecno lógica y humanística. Bussiness Support Anet
Sánchez, H. (2001). Acerca de la comprensión de lectura en educación superior. URP.

Serna, J. y Díaz, J. E. (2015). Propuesta didáctica para la comprensión. Universidad La Gran Colombia. Cuadernos de lingüística Hispana, (25), 165-180. https://doi.org/10.19053/012 1053X.3376

Smith, C. (1998). La enseñanza de la lecto-escritura: un enfoque interactivo. Aprendizaje Visor.

Solé, I. (1994). Estrategias de comprensión lectora. Grao.

Solé, I. (2004). Estrategias de lectura. Grao

Tamayo, M. (2002). El proceso de la investigación cientifica. Trillas.

Taylor (2000). Manual de lectura rápida. Noguer didáctica.

Villasante, A. (2018). Fluidez lectora en estudiantes de segundo grado de las instituciones educativas del nivel primario 70581, Acomocco y 80621 de Juliaca. https://reposito rio.upeu.edu.pe/bitstream/handle/UPEU/564/ Adela_Tesis_Licenciatura_2018.pdf?sequenc $\mathrm{e}=1$ \&isAllowed $=\mathrm{y}$ 